\title{
Pullback attractor for a non-linear evolution equation in elasticity
}

\author{
Tomás Caraballo ${ }^{\mathrm{a}}$, Renato Colucci ${ }^{\mathrm{b}}$ \\ ${ }^{a}$ Departamento de Ecuaciones Diferenciales y Análisis Numérico, Universidad de Sevilla, \\ Apdo. de Correos 1160, 41080 Sevilla, Spain \\ ${ }^{b}$ Departamento de Matemáticas \\ Pontificia Universidad Javeriana
}

Cra. 7 No. 43-82, Bogotá, Colombia

\begin{abstract}
We prove the existence of a pullback attractor for a non-autonomous fourth order evolution equation arising in the field of phase transitions and elasticity theory. The existence of several families of bounded absorbing sets is first proved in several spaces, and owing to the compactness of some inclusions between Sobolev spaces, we can then ensure the existence of a family of compact absorbing sets in the pullback sense and, as a consequence, the existence of a pullback attractor.
\end{abstract}

Key words: Pullback attractor, phase transitions, pullback absorbing family, two-parameter semigroup.

2000 MSC: 35B41, 82B26

\section{Introduction}

In [10], [11] and [12] it has been studied the asymptotic behavior of the following evolution equation:

$$
\left\{\begin{array}{l}
u_{t}=-\varepsilon^{2} u_{x x x x}+\frac{1}{2} W^{\prime \prime}\left(u_{x}\right) u_{x x} \\
u=u_{x x}=0, \quad \text { on } \partial I \\
u(0, t)=u_{0}
\end{array}\right.
$$

Email addresses: caraball@us.es (Tomás Caraballo), renatocolucci@hotmail.com (Renato Colucci)

Partly supported by FEDER and Ministerio de Economía y Competitividad (Spain) under grant MTM2011-22411. 
where $u: I \rightarrow \mathbb{R}, 0<\varepsilon<<1, I=(0,1)$ and $W(p)=\left(p^{2}-1\right)^{2}$ is the so called double well potential. The equation (1.1) represents the $L^{2}$-gradient dynamics of the non convex energy:

$$
F_{\varepsilon}(u)=\frac{1}{2} \varepsilon^{2} \int_{I} u_{x x}^{2} d x+\frac{1}{2} \int_{I} W\left(u_{x}\right) d x .
$$

The equation arises in the field of elasticity theory, phase transition and image processing.

The functional (1.2) with $\varepsilon=0$ is a simple model describing microstructures that arise from solid-solid phase transitions in certain elastic crystals (see [18] and [19]) such as In-Th Cu-Al-Ni Ni-Ti. These materials present a variety of microstructures which are important for technological application in the context of material theory (shape memory effects, pseudoelasticity, etc). From a physical point of view, the functional (1.2)(with $\varepsilon=0$ ) describes the elastic energy required for deformation of the crystal, while the function $W(\cdot)$ is the stored energy density functions describing the properties of the material. The add of the regularizing term, depending on the second derivative of $u$ and on the small parameter $\varepsilon$, solve the problem of non-uniqueness of minimizers (and of the ill-posedness of the equation of the associated $L^{2}$ gradient dynamics) without changing the relevant macroscopic features of the model. It is natural to consider the associated dynamical problem (see [1] and [2]), that is, the study of the gradient flow of the energy (1.2) which can be written in the form of system (1.1).

In [3] they studied the global dynamics of (1.1). By numerical experiments they show the existence of three well separated time scales with peculiar dynamical behavior. In a first time scale of order $t>T_{\varepsilon}=O\left(\varepsilon^{2}\right)$ the energy of the initial data is drastically reduced and microstructures (see [18]) appears in the regions where $u_{x}$ falls in the non convex region of $W$. In a second time scale of order $t>T=O(1)$ the equation exhibits a heath equation-like behavior in the regions without microstructures, while the dynamic is slow in the regions with microstructures. In the third time scale $t>\frac{1}{T_{\varepsilon}}=O\left(\varepsilon^{-2}\right)$ the equation shows a finite dimensional character, i.e. the solutions are approximately the union of consecutive segments. In particular the existence of an inertial manifold has been conjectured in [3]. In the papers [10], [11] the finite dimensional reduction of the system has been proved. In particular the authors prove the existence, giving an estimate of the dimension, of the global attractor, exponential attractor and inertial manifold. 
Nonlinear dynamical systems are subjected to aleatoric influences, that is the reason why in the present paper we consider a small non-autonomous perturbation of equation (1.1) and consider the effects on the dynamics and in particular on the third time scale.

The theory of global attractors has been generalized in the case of nonautonomous systems by introducing the concept of uniform attractor (see, for instance Chepyzhov and Vishik [9]). However this concept of uniform attractor lacks of the property of invariance which may be a serious inconvenience in the analysis of the long time behavior of the system. For this reason, an alternate possibility has been developed within the framework of pullback attractors theory (see Kloeden and Rassmussen [17], Carvalho et al. [8] and some other papers included in their bibliography sections). One advantage of the latter is that it is not necessary to impose very restrictive assumptions on the time dependent terms in the equation. Another advantage of this theory comes from the fact that, originally, the concept of pullback attractor was introduced in the field of random dynamical systems (see Schmalfuss [22], Crauel and Flandoli [14]), and for this reason it is a suitable concept to treat random and non-autonomous features in the models.

The study of non-autonomous or stochastic perturbation for fourth order evolution equation is quite recent. An important fourth order PDE is the Cahn-Hilliard equation that describes phase transitions in a binary metal alloy. In [21] the author studied the existence of an exponential attractor for the viscous Cahn-Hilliard equation:

$$
\frac{\partial}{\partial t}[v+\varepsilon(-\triangle v)]+\triangle^{2} v-\triangle W(v)=m(t),
$$

where the non-autonomous perturbation satisfies

$$
\int_{I} m(t) d x=0
$$

Stochastic perturbation have also been studied. For instance, in [7] it is considered a stochastic perturbation of the type:

$$
v_{t}+\triangle(\triangle v-W(u))=\sigma(u) \dot{B}_{t}
$$

proving the existence and uniqueness of solutions. $B_{t}$ represents a Wiener process and $\sigma$ is a bounded Lipschitz function. In the article [15] they showed 
the existence of a random attractor for a stochastic Cahn-Hilliard equation with dynamics and stochastic boundary conditions, while the stochastic three-dimensional Lagrangian averaged Navier-Stokes equations are analyzed in [6].

These arguments suggest the importance of considering perturbation of equation (1.1), in particular there is a deep relation between Cahn Hilliard equation and equation (1.1), in fact if $v$ is the solution of the Cahn-Hilliard equation:

$$
v_{t}+\triangle\left[\varepsilon^{2} \triangle v-W^{\prime}(v)\right]=0,
$$

with Neumann boundary condition

$$
\frac{\partial}{\partial n} v=\frac{\partial}{\partial n} \triangle v=0, \quad x \in\{0,1\}
$$

then

$$
u(x)=\int_{0}^{x} v(s) d s,
$$

is the solution of equation (1.1) with boudary conditions

$$
u=u_{x x}=0, \quad x \in\{0,1\} .
$$

The rest of the paper is organized as follows: in section 2 we present some useful preliminary estimates and remarks, while section 3 contains the main results, that is, the existence of several absorbing sets and the existence of a pullback attractor in $L^{2}(I)$.

\section{Preliminary estimates and definitions}

We consider a non-autonomous perturbation of the problem (1.1)

$$
\left\{\begin{array}{l}
u_{t}=-\varepsilon^{2} u_{x x x x}+\frac{1}{2} W^{\prime \prime}\left(u_{x}\right) u_{x x}+f(x, t), \quad t>\tau, x \in I, \\
u=u_{x x}=0, \quad \text { on } \partial I \\
u(\tau, x)=u_{0}(x), x \in I
\end{array}\right.
$$

where $f \in L_{\text {loc }}^{2}\left(\mathbb{R} ; L^{2}(I)\right)$ satisfies

$$
\int_{-\infty}^{t} e^{\sigma s}\left(\|f(s)\|^{2}+\left\|f^{\prime}(s)\right\|^{2}\right) d s<\infty, \quad \forall t \in \mathbb{R},
$$


for some $\sigma \in\left(0, \frac{\varepsilon^{2}}{2}\right), u_{0} \in L^{2}(I)$, and $\|\cdot\|$ is the norm of the space $L^{2}(I)$, where by $f^{\prime}(t)$ we denote $\frac{\partial f}{\partial t}(t, x)$.

We will carry on our analysis in the following spaces

$$
H=L^{2}(I), \quad V=H^{2}(I) \cap H_{0}^{1}(I),
$$

and we will denote by $u\left(\cdot ; \tau, u_{0}\right)$ the solution of $(2.1)$ corresponding to the initial datum $u_{0} \in H$ at time $\tau$. Since $|I|=1$ we have that for all $u \in V$ :

$$
\|u\| \leq\left\|u_{x}\right\| \leq\left\|u_{x x}\right\|
$$

Although, for simplicity, we will work with the interval $I=[0,1]$, we remark that our analysis is also valid, with suitable modifications, for any bounded interval $I=[a, b]$. For this reason, we will keep denoting by $|I|$ the length of this interval instead of writing 1 in what follows.

Using a slight modification of the classical results in the autonomous framework, mainly of the Faedo-Galerkin method (see [24]), we can establish the following result (see [23] or Theorems 3.1 and 3.3 in [24]; see also [10]):

Theorem 2.1. Suppose $f \in L_{l o c}^{2}(\mathbb{R} ; H)$. The problem (2.1) admits a unique solution $u$ such that, for any $T>\tau$, it follows:

$$
\begin{array}{ll}
\text { if } u_{0}=u(\tau) \in H & \Rightarrow u \in C^{0}([\tau, T], H) \cap L^{2}(\tau, T ; V) ; \\
\text { if } u_{0}=u(\tau) \in V & \Rightarrow u \in C^{0}([\tau, T], V) \cap L^{2}(\tau, T ; D(A)) ;
\end{array}
$$

where $D(A)=\left\{u \in H^{4}(I): \quad u=u_{x x}=0\right.$ in $\left.\partial I\right\}$ is the domain of the linear operator $A=\frac{\partial^{4}}{\partial x^{4}}$.

Remark 2.2. As in the autonomous case (see [10]) it is possible to prove that the solution is Lipschitz continuous with respect to initial data.

We anticipate here the calculations that uses the estimates of the $L^{2}$ norms of $u, u_{x}$ and $u_{x x}$ (see next section for details).

Indeed, let $w(\cdot)=u_{1}(\cdot)-u_{2}(\cdot)$ be the difference of two solutions associated to the initial data $u_{i}(\tau)=u_{\tau, i}, i=1,2$, i.e. $u_{i}(\cdot)=u\left(\cdot ; \tau, u_{\tau, i}\right)$. Consider the equation fulfilled by $w$, multiply it by $w$, and integrate over $I$ :

$$
\frac{1}{2} \frac{d}{d t}\|w\|^{2}+\varepsilon^{2}\left\|w_{x x}\right\|^{2}+\frac{1}{2} \int_{I}\left[W^{\prime}\left(u_{1 x}\right)-W^{\prime}\left(u_{2 x}\right)\right] w_{x} d x=0
$$


from which

$$
\begin{aligned}
\frac{1}{2} \frac{d}{d t}\|w\|^{2}+\varepsilon^{2}\left\|w_{x x}\right\|^{2} & \leq 2\left\|w_{x}\right\|^{2}-2 \int_{I} w_{x}^{2} \bar{v} d x \\
& \leq 2(1+K(t))\|w\|\left\|w_{x x}\right\| \\
& \leq \frac{(1+K(t))}{\varepsilon^{2}}\|w\|^{2}+\varepsilon^{2}\left\|w_{x x}\right\|^{2} .
\end{aligned}
$$

Then, integrating in $(\tau, t)$ with respect to time, we deduce:

$$
\left\|u_{1}-u_{2}\right\| \leq e^{\frac{1}{\varepsilon^{2}} \int_{\tau}^{t}(1+K(s)) d s}\left\|u_{\tau, 1}-u_{\tau, 2}\right\| .
$$

where $\bar{v}=u_{1 x} u_{2 x}$ and we have used (see the next section for the detailed expressions of the functions involved in the computation)

$$
\|\bar{v}\|_{\infty} \leq\left\|u_{1 x}\right\|_{\infty}\left\|u_{2 x}\right\|_{\infty} \leq\left\|u_{1 x}\right\|\left\|u_{2 x}\right\|\left\|u_{1 x x}\right\|\left\|u_{2 x x}\right\| \leq u_{\varepsilon}(t) v_{\varepsilon}(t):=K(t) .
$$

We recall now some definitions and theoretical results that will be useful in the following analysis.

Definition 2.3. A two-parameter semigroup or process $S(\cdot, \cdot)$ on a metric space $X$ is a family of maps $S(t, \tau): X \rightarrow X$, with $-\infty<\tau \leq t<\infty$ such that:

(a) $S(\tau, \tau)=I d, \forall \tau \in \mathbb{R}$,

(b) $S(t, \tau)=S(t, s) S(s, \tau) \forall \tau \leq s \leq t$.

Definition 2.4. The family of subsets of $X,\{B(t)\}_{t \in \mathbb{R}}$, is said to be pullback absorbing with respect to the process $S$ if for all $t \in \mathbb{R}$ and any bounded subset $D \subset X$, there exists $T(D, t) \leq t$ such that

$$
S(t, \tau) D \subset B(t) \text { for all } \tau \leq T(D, t) .
$$

Definition 2.5. A family of compact sets $\mathcal{A}=\{\mathcal{A}(t)\}_{t \in \mathbb{R}}$ is said to be a pullback attractor for the process $S$ if it satisfies

(a) $S(t, \tau) \mathcal{A}(\tau)=\mathcal{A}(t), \forall t \geq \tau$ (invariance property),

(b) $\lim _{\tau \rightarrow-\infty} \operatorname{dist}(S(t, \tau) D, \mathcal{A}(t))=0$ for all bounded sets $D \subset X$ (pullback attracting property).

We establish the following theorem (see [5]): 
Theorem 2.6. Let $S(t, \tau)$ be a two-parameter semigroup on a complete metric space $X$, and suppose $S(t, \tau): X \rightarrow X$ is continuous for all $t \geq \tau$. If there exists a family of compact (pullback) absorbing sets $\{B(t)\}_{t \in \mathbb{R}}$, then there exists a pullback attractor $\{\mathcal{A}(t)\}_{t \in \mathbb{R}}$, and $\mathcal{A}(t) \subset B(t)$ for all $t \in \mathbb{R}$. Furthermore,

$$
\mathcal{A}(t)=\overline{\bigcup_{\substack{D \subset X \\ \text { bounded }}} \Lambda_{D}(t)},
$$

where

$$
\Lambda_{D}(t)=\bigcap_{n \in \mathbb{N}} \overline{\bigcup_{s \geq n} S(t, t-s) D},
$$

denotes the omega limit set associated to D.

Remark 2.7. It is worth emphasizing that it is also possible to choose a more general framework for our analysis in which the attracted sets are time dependent families of sets belonging to certain universe of sets (see, for instance Caraballo et al. [4] for a detailed analysis of this situation). However, for our interest in the present paper, the situation described above is enough.

We conclude this section by recalling an important lemma that will be useful later (see [24] pag. 91).

Lemma 2.8. (The Uniform Gronwall Lemma). Let $y, g$, h three positive locally integrable functions on $\left(t_{0},+\infty\right)$ such that $y^{\prime}$ is locally integrable on $\left(t_{0},+\infty\right)$ which satisfy

$$
\begin{gathered}
\frac{d}{d t} y \leq g y+h, \quad \forall t \geq t_{0}, \\
\int_{t}^{t+r} g(s) d s \leq a_{1}, \quad \int_{t}^{t+r} h(s) d s \leq a_{2}, \quad \int_{t}^{t+r} y(s) d s \leq a_{3}, \text { for } t \geq t_{0},
\end{gathered}
$$

where $r, a_{1}, a_{2}, a_{3}$ are positive constants. Then

$$
y(t+r) \leq\left(a_{2}+\frac{a_{3}}{r}\right) e^{a_{1}}, \quad \forall t \geq t_{0} .
$$




\section{Existence of Pullback Attractor}

Thanks to Theorem 2.1 we easily deduce that our problem (2.1) generates a process $S$ in $H$ which is given by

$$
S(t, \tau) u_{0}=u\left(t ; \tau, u_{0}\right), \text { for all } t \geq \tau, u_{0} \in H
$$

Moreover, the discussion in Remark 2.2 ensures that $S(t, \tau): H \rightarrow H$ is a continuous mapping for any $t \geq \tau$. Consequently, to prove the existence of a pullback attractor for our model, we need to prove that there exists a compact (pullback) absorbing family $\{B(t)\}_{t \in \mathbb{R}} \subset H$. We will first prove the existence of an absorbing family of bounded sets in $H$. Afterwards, we will construct another family of bounded absorbing sets in $V$ and $D(A)$ which, jointly with the compact injections of these two spaces in $H$, will ensure the existence of the compact absorbing family in $H$.

Proposition 3.1. Suppose $f$ satisfies (2.2). Then, for any bounded subset $D \subset H$ and for any $t \in \mathbb{R}$, there exists $\tau_{H}(D, t) \leq t$ such that

$$
\left\|S(t, \tau) u_{0}\right\|^{2} \leq 1+2 e^{-\sigma t} \int_{-\infty}^{t}\left(\frac{1}{2 \varepsilon^{2}} e^{\sigma s}\|f(s)\|^{2}+e^{\sigma t}|I|\right) d s:=h_{\varepsilon}(t)^{2}
$$

for any $\tau \leq \tau_{H}(D, t)$ and $u_{0} \in D$.

Proof. Let us fix $t \in \mathbb{R}$ and $D \subset H$ bounded. Denote $u(\cdot)=u\left(\cdot ; \tau, u_{0}\right)$, the solution of our equation (2.1) which takes the initial value $u_{0} \in D$ at time $\tau(\leq t)$, i.e. $u(r)=S(r, \tau) u_{0}$ for all $r \in[\tau, t]$.

Throughout this proof and the rest of the paper, we will omit the time variables for simplicity in the calculations when no confusion is possible.

Multiplying equation (2.1) by $u$ we obtain

$$
\begin{aligned}
\frac{1}{2} \frac{d}{d r}\|u\|^{2}+\varepsilon^{2}\left\|u_{x x}\right\|^{2} & \leq|I|+\frac{1}{2 \varepsilon^{2}}\|f\|^{2}+\frac{\varepsilon^{2}}{2}\|u\|^{2} \\
& \leq|I|+\frac{1}{2 \varepsilon^{2}}\|f\|^{2}+\frac{\varepsilon^{2}}{2}\left\|u_{x x}\right\|^{2},
\end{aligned}
$$

from which, using (2.3), we have

$$
\frac{1}{2} \frac{d}{d r}\|u\|^{2}+\frac{\varepsilon^{2}}{2}\|u\|^{2} \leq|I|+\frac{1}{2 \varepsilon^{2}}\|f\|^{2} .
$$


Since $\sigma \in\left(0, \varepsilon^{2}\right)$,

$$
\begin{aligned}
\frac{1}{2} \frac{d}{d r}\left(e^{\sigma r}\|u(r)\|^{2}\right) & =\frac{1}{2} \sigma e^{\sigma r}\|u\|^{2}+\frac{1}{2} e^{\sigma r} \frac{d}{d r}\|u(r)\|^{2} \\
& \leq\left(\frac{\sigma}{2}-\frac{\varepsilon^{2}}{2}\right) e^{\sigma r}\|u(r)\|^{2}+e^{\sigma r}|I|+\frac{1}{2 \varepsilon^{2}} e^{\sigma r}\|f(r)\|^{2},
\end{aligned}
$$

and, integrating the previous inequality over $(\tau, t)$,

$$
e^{\sigma t}\|u(t)\|^{2} \leq e^{\sigma \tau}\left\|u_{0}\right\|^{2}+2 \int_{-\infty}^{t}\left(\frac{1}{2 \varepsilon^{2}} e^{\sigma s}\|f(s)\|^{2}+e^{\sigma t}|I|\right) d s,
$$

whence

$$
\left\|S(t, \tau) u_{0}\right\|^{2} \leq e^{\sigma(\tau-t)}\left\|u_{0}\right\|^{2}+2 e^{-\sigma t} \int_{-\infty}^{t}\left(\frac{1}{2 \varepsilon^{2}} e^{\sigma s}\|f(s)\|^{2}+e^{\sigma t}|I|\right) d s .
$$

As $D \subset H$ is bounded, there exists $d>1$ such that $\left\|u_{0}\right\| \leq d$ for all $u_{0} \in D$. Thus,

$$
\left\|S(t, \tau) u_{0}\right\|^{2} \leq e^{\sigma(\tau-t)} d^{2}+2 e^{-\sigma t} \int_{-\infty}^{t}\left(\frac{1}{2 \varepsilon^{2}} e^{\sigma s}\|f(s)\|^{2}+e^{\sigma t}|I|\right) d s,
$$

for any $u_{0} \in D$, and we conclude the proof by setting $\tau_{H}(D, t)=t-\frac{2}{\sigma} \ln d$.

We now establish an inequality that will be useful later on. We write again equation (3.1) in the following way:

$$
\frac{1}{2} \frac{d}{d t}\|u\|^{2}+\frac{\varepsilon^{2}}{2}\left\|u_{x x}\right\|^{2} \leq|I|+\frac{1}{2 \varepsilon^{2}}\|f\|^{2},
$$

and setting $\sigma \in\left(0, \varepsilon^{2}\right)$ we deduce

$$
\frac{1}{2} \frac{d}{d r}\left(e^{\sigma r}\|u\|^{2}\right)+\left(\frac{\varepsilon^{2}}{2}-\frac{\sigma}{2}\right) e^{\sigma r}\left\|u_{x x}\right\|^{2} \leq|I| e^{\sigma r}+\frac{1}{2 \varepsilon^{2}}\|f\|^{2} e^{\sigma r} .
$$

If $\tau<t-1$, we have that for all $r \in[\tau, t-1]$ :

$$
\begin{gathered}
\frac{1}{2} e^{\sigma(r+1)}\|u(r+1)\|^{2}+\left(\frac{\varepsilon^{2}}{2}-\frac{\sigma}{2}\right) \int_{r}^{r+1} e^{\sigma s}\left\|u_{x x}\right\|^{2} d s \\
\leq \frac{1}{2} e^{\sigma r}\|u(r)\|^{2}+\int_{r}^{r+1}\left(|I| e^{\sigma s}+\frac{1}{2 \varepsilon^{2}}\|f(s)\|^{2} e^{\sigma s}\right) d s \\
\leq \frac{1}{2} e^{\sigma \tau}\left\|u_{0}\right\|^{2}+\int_{-\infty}^{t}\left(|I| e^{\sigma t}+\frac{1}{2 \varepsilon^{2}}\|f(s)\|^{2} e^{\sigma s}\right) d s
\end{gathered}
$$


and then

$$
\begin{aligned}
& \int_{r}^{r+1} e^{\sigma s}\left\|u_{x x}\right\|^{2} d s \\
& \quad \leq A(\varepsilon, \sigma)\left\{e^{\sigma \tau}\left\|u_{0}\right\|^{2}+2 \int_{-\infty}^{t}\left(|I| e^{\sigma t}+\frac{1}{2 \varepsilon^{2}}\|f(s)\|^{2} e^{\sigma s}\right) d s\right\},
\end{aligned}
$$

where

$$
A(\varepsilon, \sigma):=\frac{1}{\frac{\varepsilon^{2}}{2}-\frac{\sigma}{2}} .
$$

From inequality (2.3) we obtain the same estimates for $\int_{r}^{r+1} e^{\sigma s}\|u(s)\|^{2} d s$ and $\int_{r}^{r+1} e^{\sigma s}\left\|u_{x}(s)\right\|^{2} d s$.

Moreover, denoting by $\|\cdot\|_{4}$ the norm in the space $L^{4}(I)$, we can rewrite (3.1) in a different way:

$$
\frac{d}{d t}\|u\|^{2}+4\left\|u_{x}\right\|_{4}^{4} \leq 4\left\|u_{x}\right\|^{2}+2\|f\|\|u\|
$$

from which

$$
\frac{d}{d r}\left\{e^{\sigma r}\|u\|^{2}\right\}+4 e^{\sigma r}\left\|u_{x}\right\|_{4}^{4} \leq(\sigma+1) e^{\sigma r}\|u\|^{2}+4 e^{\sigma r}\left\|u_{x}\right\|^{2}+e^{\sigma r}\|f\|^{2},
$$

and integrating in the interval $(r, r+1)$ with $r \in[\tau, t-1]$, we obtain

$$
\begin{aligned}
& e^{\sigma(r+1)}\|u(r+1)\|^{2}+4 \int_{r}^{r+1} e^{\sigma s}\left\|u_{x}(s)\right\|_{4}^{4} d s \\
& \leq\left(\frac{\varepsilon^{2}}{2}+5\right) \int_{r}^{r+1} e^{\sigma s}\left\|u_{x}(s)\right\|^{2} d s+\int_{r}^{r+1} e^{\sigma s}\|f(s)\|^{2} d s .
\end{aligned}
$$

In conclusion, we have the following estimate

$$
\begin{aligned}
\int_{r}^{r+1} e^{\sigma s}\left\|u_{x}(s)\right\|_{4}^{4} d s \leq & \left(\frac{\varepsilon^{2}}{8}+\frac{5}{4}\right) \int_{r}^{r+1} e^{\sigma s}\left\|u_{x}(s)\right\|^{2} d s \\
& +\frac{1}{4} \int_{r}^{r+1} e^{\sigma s}\|f(s)\|^{2} d s
\end{aligned}
$$


and, consequently,

$$
\begin{aligned}
\frac{1}{2} \int_{r}^{r+1} e^{\sigma s}\left(\int_{I} W\left(u_{x}\right) d x\right) d s \leq & \frac{1}{2} \int_{r}^{r+1} e^{\sigma s}\left(\left\|u_{x}\right\|_{4}^{4}+|I|\right) d s \\
\leq & \left(\frac{\varepsilon^{2}}{16}+\frac{5}{8}\right) \int_{r}^{r+1} e^{\sigma s}\left\|u_{x}(s)\right\|^{2} d s \\
& +\frac{1}{2} \int_{r}^{r+1}\left(e^{\sigma s}|I|+\frac{1}{4}\|f(s)\|^{2} e^{\sigma s}\right) d s
\end{aligned}
$$

Proposition 3.2. Suppose $f$ satisfies (2.2). Then, for any bounded subset $D \subset H$, and for any $t \in \mathbb{R}$, there exist $\tau_{U}(D, t) \leq t$ and a constant $C_{\varepsilon}>0$, such that

$$
\begin{aligned}
\left\|S(t, \tau) u_{0}\right\|_{H_{0}^{1}(I)}^{2} \leq & 1+2 C_{\varepsilon} e^{-\sigma t} \int_{-\infty}^{t}\left(|I| e^{\sigma t}+\frac{1}{2 \varepsilon^{2}}\|f(s)\|^{2} e^{\sigma s}\right) d s \\
& +\varepsilon^{2} e^{-\sigma t} \int_{-\infty}^{t} e^{\sigma s}\left\|f^{\prime}(s)\right\| d s:=u_{\varepsilon}(t)^{2}
\end{aligned}
$$

for any $\tau \leq \tau_{U}(D, t)$ and all $u_{0} \in D$.

Proof. We multiply equation (2.1) by $u_{x x}$ and obtain

$$
\begin{aligned}
\frac{1}{2} \frac{d}{d t}\left\|u_{x}\right\|^{2}+\varepsilon^{2}\left\|u_{x x x}\right\|^{2} & =-\frac{1}{2}\left\langle\left[W^{\prime}\left(u_{x}\right)\right]_{x}, u_{x x}\right\rangle-\left\langle f(t), u_{x x}\right\rangle \\
& =\left\langle 2-6 u_{x}^{2}, u_{x x}^{2}\right\rangle+\left\langle f^{\prime}(t), u_{x}\right\rangle \\
& \leq\left\|u_{x x}\right\|^{2}+\left\|f^{\prime}(t)\right\|\left\|u_{x}\right\| \\
& \leq \frac{1}{4 \varepsilon^{2}}\left\|u_{x}\right\|^{2}+\varepsilon^{2}\left\|u_{x x x}\right\|^{2}+\frac{\varepsilon^{2}}{2}\left\|f^{\prime}(t)\right\|^{2}+\frac{1}{2 \varepsilon^{2}}\left\|u_{x}\right\|^{2}
\end{aligned}
$$

from which we deduce

$$
\frac{d}{d t}\left\|u_{x}\right\|^{2} \leq \frac{3}{2 \varepsilon^{2}}\left\|u_{x}\right\|^{2}+\varepsilon^{2}\left\|f^{\prime}(t)\right\|^{2} .
$$

Then, using the same argument of the previous proposition, we have:

$$
\frac{d}{d r}\left(e^{\sigma r}\left\|u_{x}\right\|^{2}\right) \leq\left(\frac{3}{2 \varepsilon^{2}}+\frac{\varepsilon^{2}}{2}\right) e^{\sigma r}\left\|u_{x}\right\|^{2}+\varepsilon^{2} e^{\sigma r}\left\|f^{\prime}(r)\right\|^{2} .
$$


As $D \subset H$ is bounded, there exists $d>1$ such that $\left\|u_{0}\right\| \leq d$ for all $u_{0} \in D$. Then if $\tau<t-1$ for all $r \in[\tau, t-1]$ we obtain the following inequality by using the Uniform Gronwall Lemma

$$
\begin{gathered}
e^{\sigma(r+1)}\left\|u_{x}(r+1)\right\|^{2} \\
\leq e^{\frac{3}{2 \varepsilon^{2}}+\frac{\varepsilon^{2}}{2}}\left\{\int_{r}^{r+1} e^{\sigma s}\left\|u_{x}(s)\right\| d s\right\}+\varepsilon^{2} \int_{r}^{r+1} e^{\sigma s}\left\|f^{\prime}(s)\right\| d s, \\
e^{\sigma t}\left\|u_{x}(t)\right\|^{2} \\
\leq A(\varepsilon, \sigma)\left(e^{\frac{3}{2 \varepsilon^{2}}+\frac{\varepsilon^{2}}{2}}\right)\left\{e^{\sigma \tau} d^{2}+2 \int_{-\infty}^{t}\left(|I| e^{\sigma t}+\frac{1}{2 \varepsilon^{2}}\|f(s)\|^{2} e^{\sigma s}\right) d s\right\} \\
+\varepsilon^{2} \int_{-\infty}^{t} e^{\sigma s}\left\|f^{\prime}(s)\right\| d s,
\end{gathered}
$$

from which

$$
\begin{aligned}
& \left\|S(t, \tau) u_{0}\right\|_{H_{0}^{1}(I)}^{2} \\
& \leq C_{\varepsilon}\left\{e^{\sigma(\tau-t)} d^{2}+2 e^{-\sigma t} \int_{-\infty}^{t}\left(|I| e^{\sigma t}+\frac{1}{2 \varepsilon^{2}}\|f(s)\|^{2} e^{\sigma s}\right) d s\right\} \\
& +\varepsilon^{2} e^{-\sigma t} \int_{-\infty}^{t} e^{\sigma s}\left\|f^{\prime}(s)\right\| d s
\end{aligned}
$$

where

$$
C_{\varepsilon}=A(\varepsilon, \sigma)\left(e^{\frac{3}{2 \varepsilon^{2}}+\frac{\varepsilon^{2}}{2}}\right)
$$

We conclude the proof by setting $\tau_{U}(D, t)=t-\frac{1}{\sigma} \ln \left(C_{\varepsilon} d^{2}\right)$.

Multiplying again equation (2.1) by $u_{x x}$ :

$$
\frac{1}{2} \frac{d}{d t}\left\|u_{x}\right\|^{2}+\varepsilon^{2}\left\|u_{x x x}\right\|^{2} \leq\left\|u_{x x}\right\|^{2}+\left\|f^{\prime}\right\|\left\|u_{x}\right\|
$$

and, consequently,

$$
\frac{1}{2} \frac{d}{d t}\left\|u_{x}\right\|^{2}+\frac{\varepsilon^{2}}{2}\left\|u_{x x x}\right\|^{2} \leq \frac{1}{\varepsilon^{2}}\left\|u_{x}\right\|^{2}+\frac{\varepsilon^{2}}{2}\left\|f^{\prime}\right\|^{2} .
$$

We then have:

$$
\frac{d}{d r}\left(e^{\sigma r}\left\|u_{x}\right\|^{2}\right)-\sigma e^{\sigma r}\left\|u_{x}\right\|^{2}+\varepsilon^{2} e^{\sigma r}\left\|u_{x x x}\right\|^{2} \leq \frac{2}{\varepsilon^{2}} e^{\sigma r}\left\|u_{x}\right\|^{2}+\varepsilon^{2} e^{\sigma r}\left\|f^{\prime}\right\|^{2} .
$$


Integrating in $(r, r+1)$,

$$
\begin{array}{r}
e^{\sigma(r+1)}\left\|u_{x}(r+1)\right\|^{2}-e^{\sigma r}\left\|u_{x}(r)\right\|^{2}+\varepsilon^{2} \int_{r}^{r+1} e^{\sigma s}\left\|u_{x x x}\right\|^{2} d s \\
\leq\left(\frac{2}{\varepsilon^{2}}+\frac{\varepsilon^{2}}{2}\right) \int_{r}^{r+1} e^{\sigma s}\left\|u_{x}\right\|^{2} d s+\varepsilon^{2} \int_{r}^{r+1} e^{\sigma s}\left\|f^{\prime}\right\|^{2} d s .
\end{array}
$$

Thus

$$
\begin{aligned}
\int_{r}^{r+1} e^{\sigma s}\left\|u_{x x x}\right\|^{2} d s \leq & \frac{1}{\varepsilon^{2}}\left\{e^{\sigma r}\left\|u_{x}(r)\right\|^{2}+\left(\frac{2}{\varepsilon^{2}}+\frac{\varepsilon^{2}}{2}\right) \int_{r}^{r+1} e^{\sigma s}\left\|u_{x}\right\|^{2} d s\right. \\
& \left.+\varepsilon^{2} \int_{r}^{r+1} e^{\sigma s}\left\|f^{\prime}\right\|^{2} d s\right\}
\end{aligned}
$$

whence

$$
\begin{aligned}
\int_{r}^{r+1} & e^{\sigma s}\left\|u_{x x x}\right\|^{2} d s \\
\leq & {\left[\frac{C_{\varepsilon}}{\varepsilon^{2}}+\frac{2}{\varepsilon^{4}}+\frac{1}{2}\right]\left[e^{\sigma \tau}\left\|u_{0}\right\|^{2}+2 \int_{-\infty}^{t}\left(|I| e^{\sigma t}+\frac{1}{2 \varepsilon^{2}}\|f(s)\|^{2} e^{\sigma s}\right) d s\right] } \\
& +2 \int_{-\infty}^{t} e^{\sigma s}\left\|f^{\prime}\right\|^{2} d s .
\end{aligned}
$$

Now we can state our next result.

Proposition 3.3. Suppose $f$ satisfies (2.2). Then for any bounded subset $D \subset H$, and for any $t \in \mathbb{R}$, there exists $\tau_{V}(D, t) \leq t$ and $v_{\varepsilon}(t)$ such that

$$
\left\|S(t, \tau) u_{0}\right\|_{H^{2}(I)} \leq v_{\varepsilon}(t)
$$

for any $\tau \leq \tau_{V}(D, t)$ and $u_{0} \in D$.

Proof. Multiplying equation by $u_{t}$ and integrating over $I$ yield that

$$
\left\|u_{t}\right\|^{2}+\varepsilon^{2} \int_{I} u_{x x x x} u_{t} d x=\int_{I}\left[W^{\prime}\left(u_{x}\right)\right]_{x} u_{t} d x+\int_{I} f u_{t} d x .
$$

We can write the previous inequality in the following way:

$$
\begin{aligned}
\left\|u_{t}\right\|^{2}+\frac{d}{d t}\left\{\frac{\varepsilon^{2}}{2}\left\|u_{x x}\right\|^{2}+\frac{1}{2} \int_{I} W\left(u_{x}\right) d x\right\} & =\int_{I} f u_{t} d x \\
& \leq\|f\|\left\|u_{t}\right\| \\
& \leq \frac{1}{4}\|f\|^{2}+\left\|u_{t}\right\|^{2}
\end{aligned}
$$


and, thus,

$$
\frac{d}{d t}\left\{\frac{\varepsilon^{2}}{2}\left\|u_{x x}\right\|^{2}+\frac{1}{2} \int_{I} W\left(u_{x}\right) d x\right\} \leq \frac{1}{4}\|f\|^{2} .
$$

Then, using the same arguments of the previous propositions and taking into account that $\sigma \in\left(0, \frac{\varepsilon^{2}}{2}\right)$, we can write:

$$
\begin{aligned}
\frac{d}{d r} & \left\{\frac{\varepsilon^{2}}{2} e^{\sigma r}\left\|u_{x x}\right\|^{2}+\frac{1}{2} e^{\sigma r} \int_{I} W\left(u_{x}\right) d x\right\} \\
& \leq \frac{1}{4} e^{\sigma r}\|f\|^{2}+\frac{\varepsilon^{2}}{2}\left\{\frac{\varepsilon^{2}}{2} e^{\sigma r}\left\|u_{x x}\right\|^{2}+\frac{1}{2} e^{\sigma r} \int_{I} W\left(u_{x}\right) d x\right\} .
\end{aligned}
$$

We can use the Uniform Gronwall Lemma in the interval $(r, r+1)$ with

$$
a_{1}=\frac{\varepsilon^{2}}{2}, \quad a_{2}=\frac{1}{4} \int_{-\infty}^{t} e^{\sigma s}\|f(s)\|^{2} d s,
$$

and

$$
\begin{gathered}
a_{3}=\left(\frac{9}{16} \varepsilon^{2}+\frac{5}{8}\right) A(\varepsilon, \sigma)\left[e^{\sigma \tau}\left\|u_{0}\right\|^{2}+2 \int_{-\infty}^{t}\left(|I| e^{\sigma t}+\frac{1}{2 \varepsilon^{2}} e^{\sigma s}\|f(s)\|^{2}\right) d s\right] \\
+\frac{1}{2} \int_{-\infty}^{t}\left(e^{\sigma t}|I|+\frac{1}{4}\|f(s)\|^{2} e^{\sigma s}\right) d s,
\end{gathered}
$$

where we have used inequalities (3.5), (3.7) and (3.8).

Then we obtain:

$$
\frac{\varepsilon^{2}}{2} e^{\sigma t}\left\|u_{x x}\right\|^{2}+\frac{1}{2} e^{\sigma t} \int_{I} W\left(u_{x}\right) d x \leq e^{a_{1}}\left(a_{2}+a_{3}\right) .
$$

Since $W\left(u_{x}\right)$ is non negative we get the estimate

$$
\begin{aligned}
e^{\sigma t}\left\|u_{x x}\right\|^{2} & \leq B(\varepsilon, \sigma) e^{\sigma \tau} d^{2}+2 B(\varepsilon, \sigma) \int_{-\infty}^{t}\left(e^{\sigma t}|I|+\frac{1}{2 \varepsilon^{2}}\|f(s)\|^{2} e^{\sigma s}\right) d s \\
& +\frac{e^{\frac{\varepsilon^{2}}{2}}}{\varepsilon^{2}} \int_{-\infty}^{t}\left(e^{\sigma t}|I|+\frac{1}{4}\|f(s)\|^{2} e^{\sigma s}\right) d s+\frac{e^{\frac{\varepsilon^{2}}{2}}}{2 \varepsilon^{2}} \int_{-\infty}^{t}\|f(s)\|^{2} e^{\sigma s} d s,
\end{aligned}
$$

where

$$
B(\varepsilon, \sigma):=\frac{2}{\varepsilon^{2}} e^{\frac{\varepsilon^{2}}{2}}\left(\frac{9}{16} \varepsilon^{2}+\frac{5}{8}\right) A(\varepsilon, \sigma) .
$$


Then we have

$$
\begin{aligned}
\left\|u_{x x}\left(t ; \tau, u_{0}\right)\right\|^{2} \leq & 1+2 B(\varepsilon, \sigma) e^{-\sigma t} \int_{-\infty}^{t}\left(e^{\sigma t}|I|+\frac{1}{2 \varepsilon^{2}}\|f(s)\|^{2} e^{\sigma s}\right) d s \\
& +\frac{e^{\frac{\varepsilon^{2}}{2}}}{\varepsilon^{2}} e^{-\sigma t} \int_{-\infty}^{t}\left(e^{\sigma t}|I|+\frac{1}{4}\|f(s)\|^{2} e^{\sigma s}\right) d s \\
& +\frac{e^{\frac{\varepsilon^{2}}{2}}}{2 \varepsilon^{2}} e^{-\sigma t} \int_{-\infty}^{t}\|f(s)\|^{2} e^{\sigma s} d s:=\tilde{v}_{\epsilon}^{2}(t)
\end{aligned}
$$

for all $u_{0} \in D$ and all $\tau \leq \tilde{\tau}_{V}$, where

$$
\tilde{\tau}_{V}:=t-\frac{1}{\sigma} \ln \left(B(\varepsilon, \sigma) d^{2}\right),
$$

We conclude the proof by setting

$$
v_{\varepsilon}^{2}(t):=h_{\varepsilon}^{2}(t)+u_{\varepsilon}^{2}(t)+\tilde{v}_{\varepsilon}^{2}(t),
$$

and $\left.\tau_{V}=\min \left\{\tau_{H}, \tau_{U}, \tilde{\tau}_{V}\right)\right\}$

From the above three propositions and from theorem (2.6) we deduce the following result:

Theorem 3.4. Suppose $f$ satisfies (2.2) for some $\sigma \in\left(0, \varepsilon^{2}\right)$. Then the system (2.1) possesses a pullback attractor in $L^{2}(I)$.

Proof. From the above proposition we have that the set

$B(t):=\left\{u \in H \cap H_{0}^{1}(I) \cap H^{2}(I): \quad\|u\| \leq h_{\varepsilon}(t),\left\|u_{x}\right\| \leq u_{\varepsilon}(t),\left\|u_{x x}\right\| \leq v_{\varepsilon}(t)\right\}$,

is pullback absorbing in $L^{2}(I)$. The compactness of $B(t)$ in $L^{2}(I)$ follows from the compact embedding of $H_{0}^{1}(I)$ in $L^{2}(I)$.

\section{References}

[1] Ball, J. M. Some open problems in elasticity. Geometry, mechanics, and dynamics, 359, Springer, New York, 2002 
[2] Ball, J. M.; Holmes, P. J.; James, R. D.; Pego, R. L.; Swart, P.J. On the dynamics of fine structure. J. Nonlinear Sci. 1(1991), no. 1, 1770.

[3] Bellettini, G.; Fusco, G.; Guglielmi, N. A concept of solution and numerical experiments for forward-backward diffusion equations. Discrete Continuous Dynamical Systems 16 (2006), no. 4, 783-842.

[4] Caraballo, T.; Łukaszewicz, G.; Real, J.; Pullback attractors for asymptotically compact non-autonomous dynamical systems, Nonlinear Analysis 64 (2006) 484498

[5] Caraballo, T.; Real, J., Attractors for 2D-NavierStokes models with delays. J. Differential Equations 205 (2004) 271297

[6] Caraballo, T.; Real, J.; Taniguchi, T. On the existence and uniqueness of solutions to stochastic three-dimensional Lagrangian averaged NavierStokes equations. Proc. R. Soc. A 462 (2006), 459479

[7] Cardon-Weber, C. Cahn-Hilliard stochastic equation: existence of the solution and of its density. Bernoulli 7 (2001), no. 5.

[8] Carvalho, A.N.; Langa, J.A.; Robinson, J.C.; Attractors for infinitedimensional non-autonomous dynamical systems. Applied Mathematical Sciences, 182. Springer, New York, 2013

[9] Chepyzhov, V.V.; Vishik, M.I.; Attractors for equations of mathematical physics. American Mathematical Society Colloquium Publications, 49. American Mathematical Society, Providence, RI, 2002.

[10] Colucci, R.; Chacón, G.R. Asymptotic behavior of a fourth order evolution equation. Submitted Paper.

[11] Colucci, R.; Chacón, G.R. Dimension Estimate for the Global Attractor of an evolution equation. Abstract and Applied Analysis Volume 2012, Article ID 541426, 18 pages, doi:10.1155/2012/541426.

[12] Colucci, R.; Chacón, G.R. Hyperbolic Relaxation of a Fourth Order Evolution Equation. Abstract and Applied Analysis Volume 2013, Article ID 372726, 11 pages http://dx.doi.org/10.1155/2013/372726 
[13] Constantin, P.; Foias, C.; Nicolaenko, B.; Temam, R. Integral Manifolds and Inertial Manifolds for Dissipative Partial Differential Equations. Applied Mathematical Sciences v. 70, Springer; 1 edition (October $25,1988)$.

[14] Crauel, H; Flandoli, F.; Attractors for random dynamical systems, Probability Theory and Related Fields 100(1994), 365-393.

[15] Desheng, Y.; Duan, J. An impact of stochastic dynamic boundary conditions on the evolution of the Cahn-Hilliard system. Stoch. Anal. Appl. 25 (2007), no. 3.

[16] Eden, A.; Foias, C.; Nicolaenko, B.; Temam, R. Exponential Attractors for Dissipative Evolution Equations. John Wiley \& Sons Inc (June 1995).

[17] Kloeden, P. E.; Rasmussen, M. Nonautonomous Dynamical Systems. American Mathematical Society (August 17, 2011)

[18] Müller, S. Variational models for microstructure and phase transitions. Calculus of variations and geometric evolution problems (Cetraro, 1996), 85-210, Lecture Notes in Math., 1713, Springer, Berlin, 1999.

[19] Pedregal, P. Variational methods in Nonlinear Elasticity. Society for Industrial and Applied Mathematics (SIAM), Philadelphia, PA, 2000.

[20] Robinson, J.C. Infinite-dimensional dynamical systems. An introduction to dissipative parabolic PDEs and the theory of global attractors. Cambridge Texts in Applied Mathematics. ambridge University Press, Cambridge, 2001.

[21] Saoud, B. Pullback exponential attractors for the viscous Cahn-Hilliard equation in bounded domains. Journal of Applied Analysis and Computation VOL 2, NO 1 (2012).

[22] Schmalfuss, B. Backward cocycle and attractors of stochastic differential equations, in V. Reitmann, T. Redrich and N. J. Kosch (eds.), International Seminar on Applied Mathematics-Nonlinear Dynamics: Attractor Approximation and Global Behaviour (1992), 185-192.

[23] Slemrod, M. Dynamics of measured valued solutions to a backwardforward heat equation. Journal of Dynamics and Differential Equations Volume 3, Number 1, 1-28, 1991 
[24] Temam, R. Infinite Dimensonal Dynamical Systems in Mechanics and Physics. Springer; 2nd edition (April 1, 1997). 